\title{
Genome Wide Characterization and Comparative Analysis of Simple Sequence Repeats in Cucurbita Genomes
}

\section{Lei Zhu}

Henan Agricultural University https://orcid.org/0000-0003-4210-1531

Hua yu Zhu

Henan Agricultural University

Yan man Li

Henan Agricultural University

Xiang bin Wu

Henan Agricultural University

Jin tao Li

Henan Agricultural University

Zhen li Zhang

Henan Agricultural University

Yan jiao Wang

Henan Agricultural University

Jian bin $\mathrm{Hu}$

Henan Agricultural University

Sen Yang

Henan Agricultural University

Yong qiang $\mathrm{Li}$

Henan Agricultural University

Shou ru Sun ( $\sim$ ssr365@sina.com )

Lu Ming Yang

Henan Agricultural University

Research article

Keywords: Cucurbita species, SSR, the syntenic relationship, Genetic diversity, MAS

Posted Date: July 9th, 2020

DOI: https://doi.org/10.21203/rs.3.rs-40696/v1 
License: (c) (i) This work is licensed under a Creative Commons Attribution 4.0 International License. Read Full License 


\section{Genome wide characterization and comparative analysis of simple sequence repeats in Cucurbita genomes}

Lei Zhu ${ }^{\dagger}$, Huayu Zhu ${ }^{\dagger}$, Yanman Li, Xiangbin Wu, Jintao Li, Zhenli Zhang, Yanjiao Wang, Jianbin Hu, Sen Yang, Yongqiang Li, Shouru Sun*, Luming Yang*

College of Horticulture, Henan Agricultural University, 95 Wenhua Road,

Zhengzhou, 450002, China

$\dagger$ These authors contributed equally to this work

* For correspondence email: the E-mail of the first corresponding author:

ssr365@ sina.com, the E-mail of the second corresponding author:

lumingyang@henau.edu.cn 


\begin{abstract}
Background

The Cucurbita genus contains important economic crops in the world, while limited molecular markers have been developed in the past years. Simple sequence repeats (SSR) markers are powerful tools for the study of genetic mapping construction, genetic diversity analysis and genome wide association. The availability of pumpkin genome information has made it possible to analyze SSRs in genome wide across three Cucurbita species.
\end{abstract}

\title{
Results
}

In this paper, based on the whole genome sequences, 34,375 SSR loci were found in C. moschata, 30,577 SSR loci were found in C. maxima and 38,104 SSR loci were found in C. pepo. C. pepo has the maximum density of SSRs with an average of 145 $\mathrm{SSR} / \mathrm{Mb}$. In general, the frequency in total SSR loci decreased with the increase of the motif length, dinucleotide motifs were the most common motifs in the three species, and for the same repeat types, the SSR frequency decreased sharply with the increase of the repeat number. Most of those SSR loci were suitable for marker development (84.75\% in C. moscata, $94.53 \%$ in C. maxima and $95.09 \%$ in C. pepo). Based on those markers, we compared and analyzed the cross-species SSR markers between $C$. pepo and other Cucurbitaceae species by silico-PCR. Using these cross-species primers, the high collinear relationships between $C$. pepo and the other two species were detected, respectively. Furthermore, the application of SSR markers in genetic diversity analysis was tested in C. pepo, the results showed that they were good tools to be used in genetic diversity analysis.

\section{Conclusion}

In this study, the genome wide SSR markers were detected from three Cucurbita species, and some of their applications were proved by comparative genomics and genetic diversity analysis. The large number of genome-wide SSR markers and crossspecies markers would promote the basic and applied studies of Cucurbita species, such as gene mapping, QTLs mapping, comparative genomics and marker-assisted breeding. 
Page 3

Keywords: Cucurbita species, SSR, the syntenic relationship, Genetic diversity, MAS 


\section{Background}

The Cucurbita genus $(2 \mathrm{n}=2 \times=40)$, belonging to the Cucurbitaceae family, contains 13-30 species [1]. Most of these species are wild resources, and only three domesticated species including Cucurbita. Maxima, Cucurbita. Moschata, and Cucurbita. Pepo are widely cultivated in the world [2]. The earliest archaeological evidence for the domestication of Cucurbita species was about 8,00010,000 years ago $[3,4]$. The Cucurbita genus, native to the America and southern Mexico, was firstly introduced into European and then underwent a great diversification in Asia, which later became the secondary domestication center $[3,5$, 6]. Nowadays, as an important economic crop, pumpkin has been widely grown all over the world. The largest cultivation area for pumpkin is Asia, followed by Europe and South America, and China is the main pumpkin production country in Asia. In 2012, the planting area of pumpkin in China was about $3.8 \times 10^{4} \mathrm{Hm}^{2}$, and the total output reached $7.0 \times 10^{6}$ tons (http://faostat3.fao.org/). Cucurbita species have a relatively smaller genome sizes and a greater chromosome numbers compared with other Cucurbitaceous crops [7], and the assembled genome sizes are $271.4 \mathrm{Mb}$ for $C$. maxima, 269.9 Mb for C. moschata, and $263 \mathrm{Mb}$ for $C$. pepo, respectively [8, 9]. With a long history of cultivation and domestication, the morphological variation of Cucurbita species shows a higher diversity in fruit shape, size and color than other Cucurbitaceae species [10]. Furthermore, Cucurbita species have strong roots and wide adaption to different adversities, such as cold stress, virus infection, and high salt, so they are widely used as rootstocks in grafting [11, 12].

Simple sequence repeats (SSR), because of their relative abundance, multi-allelism, co-dominance and cheap costs, are widely used in genetic mapping constructions, genetic diversity analysis and genome wide association studies [13, 14]. The whole genome sequencing of cucumber, melon and watermelon has been completed for years [15-17], and the genome-wide SSR markers in these crops have been characterized and developed, which have greatly promoted their application in gene and QTL mapping, comparative genomics [18-20]. For example, the syntenic relationships between melon and cucumber chromosomes were revealed using 199 SSR markers [21]. The genome syntenic relationships among watermelon, melon and 
cucumber were confirmed by comparative mapping [22]. Based on massive genomewide SSR markers, the complicated mosaic patterns of the chromosome synteny between melon and watermelon or cucumber were also established [23]. However, the developments of SSR markers in cucurbit species were limited. According to the conserved sequences among species and genera, a few of AFLP, RAPD and SSR markers were developed in previous studies [24-28]. These markers were still far from enough in the genetic diversity or comparative genomics researches. Although Esteras et al, constructed the first Cucurbita genetic map based on the SNPs and found that the Cucurbita linkage groups were partially homoeologous to cucumber chromosomes using 304 SNPs and 11 SSR markers [29], the applications of these EST-SNP markers still had a great limitation. For example, the high cost of enzymes and the complicated operation procedure. Until recently, the related reports about Cucurbita genus gradually increased and the whole genome sequence of pumpkin was available. The whole-genome synteny analysis indicated that both $C$. maxima and $C$. moschata genomes underwent a whole genome duplication (WGD) event and pairs of C. maxima (or C. moschata) homoeologous regions shared between chromosomes corresponding to two sub-genomes [9]. Latterly, Montero et al. analyzed the genomic synteny between $C$. pepo and other Cucurbitaceae species (Cucumis melo, Cucumis sativus and Citrullus lanatus) and identified that the covered regions in most of the zucchini genome had a whole genome duplication again [8]. Furthermore, some transcriptomes of Cucurbita species were completed, and the EST-SSRs were developed [30-34]. The successive reports of Cucurbita genomic resources greatly promoted the large-scale development of SSR markers and gave us a benefit to construct the high-resolution maps and study the syntenic relationship and chromosomal rearrangements between Cucurbita species.

In this study, we carried out a genome wide identification of SSR motifs in three Cucurbita species, analyzed the distribution and frequency of different repeats types and studied the chromosome synteny of Cucurbita pepo with other Cucuribitaceae species. The derived SSR loci will be useful for population structure, genetic diversity, molecular assisted selection, map-based cloning and other studies in Cucurbita species. 


\section{Results}

\section{The frequency and distribution of different SSR types in Cucurbita genome}

A total of 103,056 microsatellite sequences were identified in Cucurbita genome, including 34,375 SSR loci in the released $269.9 \mathrm{Mb}$ genomic sequences of $C$. moschata cv. Rifu, 30,577 SSR loci in the released 271.4 Mb genomic sequences of $C$. maxima cv. Rimu and 38,104 SSR loci in the released $263 \mathrm{Mb}$ genomic sequences of C. pepo MU-CU-16 (Table. 1). Obviously, C. pepo has the maximum density of SSRs with an average of $145 \mathrm{SSR} / \mathrm{Mb}$.

Here, we analysed repeat types ranged from dinucleotide to octanucleotide. Among all of these nucleotide motifs, dinucleotide motifs (41.0\%) seemed to be the most common motifs accounting for $41.78 \%, 39.90 \%$ and $41.01 \%$ of the total SSR loci discovered in the three genomes, respectively, followed by trinucleotide motifs (16.97\%, 19.19\%, and $17.88 \%$, respectively), the octanucleotide motifs (3.78\%, $3.76 \%$, and $3.38 \%$, respectively) were the least represented repeat types (Table. 1). In general, the frequency in total SSR loci decreased with increase of the motif length, except for heptanucleotide.

We further examined the SSR motif distribution with regard to their repeat numbers (Fig. 1). For all the repeat types, with increased of the repeat number, the SSR frequency decreased sharply, and this change was more obvious in the longer SSR motifs (Fig. 1). Consequently, the mean repeat numbers in dinucleotides were the highest in all the repeat types (Table. 1). The analysis of individual SSR types revealed that some of its own specific motifs were more prevalent than others in each class (Additional file 1, Fig S1). For example, The AT motif also was the most frequent motif in the three genomes, which accounting for $31.61 \%$ (in C. moschata), $28.81 \%$ (in C. maxima) and $30.45 \%$ (in C.pepo) of the total loci discovered. Similarly, the AAT, AAAT, AAAAT, AAAAAT, AAAAAAT, and AAAAAAAT motif (AATAATAT motif in C. maxima) were the most frequent types in each class, respectively (Additional file 1, Fig S1). These results revealed that AT-rich motifs were the most abundant ones in all SSR motifs in $C$. moschata, $C$. maxima and $C$. pepo genomes. 
We investigated the SSRfrequencies in each chromosome of the three Cucurbit species (Table. 2). The frequency of microsatellite loci was not correlated with the chromosome size. For example, in the $C$. moschata genome, the SSR density of the longest chromosome (Chr04) was just at the middle level, while Chr02having a much shorter length than Chr04 had the highest SSR density. Similar things also happened in the other two genomes indicating that the distribution of SSRs was uneven in the Chromosomes (Table. 2). For better understanding of the distributions of different SSR motifs, we further checked their frequencies on each chromosome (Fig. 2). The results showed that different SSR types had the same tendency among different chromosomes, it seemed that the distribution of different SSR types on one chromosome was decided by their frequencies in the whole genome and the SSR density of the Chromosome (Fig. 2).

The genomics sequences containing these microsatellites were screened for PCR primer design, and 94,272 SSR microsatellite loci contained suitable flanking sites for SSR primer design. C. moschata had the lowest proportion of SSRs suitable for primers design (84.75\%), while the percentages in $C$. maxima and $C$. pepo reached $94.53 \%$ and $95.09 \%$, respectively (Table. 1). Though the dinucleotide repeat type was the most frequent one in all the three genomes, it didn't have a good peformance in primers design. Hexatanucleotide repeat type has the highest ratio of SSRs suitable for primers design, followed by pentanucleotide repeat types in all the three genomes, indicating that the longer motifs were more suitable for primers design in Cucurbita (Table. 1). Finally, 91,248 SSR primers (28,194 in C. moschata, 28,061 in C. maxima and 34,993 in C. pepo) were designed with some primers included more than one SSR loci as compound SSR (Additional file 2, Table S2-Table S4).

\section{Chromosome synteny relationship of Cucurbita pepo with other Cucurbitaceae} species

In order to understand the universality and correlation of SSR markers among Cucurbitaceae crops, we compared and analyzed the cross-species SSR markers between C. pepo and other Cucurbitaceae species by silico-PCR. We identified 391 cross-species SSR markers between $C$. pepo and C. sativus, 425 cross-species SSR markers between $C$. pepo and $C$. melo, 717 cross-species SSR markers between $C$. 
pepo and C. lanatus, 11,732 cross-species SSR markers between $C$. pepo and $C$. maxima, and 15,274 cross-species SSR markers between C. pepo and C. moschata (Additional file 2, Table S5-S9). Collinear blocks to inversion-blocks ratio was 26:26 between $C$. pepo and $C$. sativus genomes, 25:36 between $C$. pepo and C. melo genomes, 51:38 between C. pepo and C. lanatus genomes, 154:158 between C. pepo and C. maxima genomes, and 153:152 between $C$. pepo and C. moschata genomes (Additional file 2, Table S5-S9). Interestingly, the ratio of collinear-blocks to inversion-blocks was nearly 1:1 among three Cucurbita species. Each C. pepo blocks shared 3-34 SSR markers with C. sativus, C. lanatus or C. melo. However, most of the $C$. pepo syntenic block shared larger number of SSR markers $(3-1,080)$ with $C$. maxima or C. moschata. The C. pepo syntenic block CpeCmos53 had the largest number of shared SSR markers $(1,080)$ between Cpe-Chr03 and Cmo-Chr14, which indicated that these regions were conservative during chromosome evolution.

By comparing the physical positions of those common shared-markers, the main syntenic relationships between $C$. pepo and other Cucurbitaceae species were listed in Table. 3, and the visualized chromosomes syntenic relationships of $C$. pepo with $C$. lanatus, C. melo, and C. sativus were shown in Fig. 3. The main syntenic relationships among chromosomes revealed complex mosaic patterns. In Fig. 3, each C. pepo chromosome was syntenic to more than two chromosomes in other Cucuribitaceae species. C. pepo chromosome Cpe9 and Cpe16 had the simplest syntenic pattern with watermelon, and each of them was mainly syntenic to one watermelon chromosome (Table. 3). Cpe9 was syntenic to watermelon chromosome W5, and 14 commonly shared SSR markers were found on them. From marker CpeSSR15544 to CpeSSR16107, there were three blocks and each block contained at least four SSR markers. According to the continuous physical positions of these markers on both of the reference genomes, the block CpeWM37 and CpeWM38 showed an inversion pattern and the block CpeWM39 showed a collinear pattern between $C$. pepo and $C$. lanatus (Aditional file 2, Table S7). Similar comparison works were carried out between $C$. pepo and $C$. sativus or $C$. pepo and C. melo using the cross-species SSR markers. C. pepo chromosomes cpe7, cpe8, cpe11 and cpe20 had the simplest syntenic pattern with $C$. sativus, while each of them just syntenic to 
one cucumber chromosome. Meanwhile, the simplest syntenic pattern between $C$. pepo and C. melo was mainly found on Cpe15, Cpe18, Cpe19 and Cpe20 (Table. 3). The most complicated syntenic pattern was found on Cpe1, which was corresponding to five chromosomes of C.moschata, four chromosomes of C. maxima, seven chromosomes of $C$. lanatus, three chromosomes of $C$. sativus and five chromosomes of C. melon (Table. 3).

Compared with the above analysis, the syntenic relationships between $C$. pepo and C. moschata, or between $C$. pepo and C. maxima were more simple and clear. Each of the 20 chromosomes in $C$. pepo was mainly syntenic with one chromosome in $C$. moschata and one chromosome in C. maxima (Table. 3). In order to see their syntenic relationships more directly, the single chromosome comparison analysis was carried out (Fig. 4). From Fig. 4, it was easy to find that each chromosome in C. pepo was collinear to one chromosome in C. moschata and one chromosome in C. maxima, these three chromosomes had highly consistency; the two chromosomes, individually from $C$. moschata and C. maxima, even were named as the same order in each genome. For example, Cpe1 was syntenic with $\mathrm{Cmo} 4$ and $\mathrm{Cma} 4, \mathrm{Cpe} 2$ was syntenic with Cmo1 and Cma1 (Fig. 4). Those results indicated that $C$. pepo had closer relationships with $C$. moschata and $C$. maxima, compared with other species in Cucurbitaceae; $C$. moschata and $C$. maxima might have a closer syntenic relationship than with C. pepo.

\section{Application of SSR markers in C.pepo genetic diversity and population structure analysis}

In a preliminary experiment, about four hundred SSR markers were screened using 61 materials. Based on the account of allelic number, the genomic coverage, and the PCR amplification efficiency, 66-core SSR markers were obtained (Additional file 2, Table S10). These markers exhibited clear band spectrums and distributed evenly on the chromosomes. In this study, 66 pairs of primers amplified 276 polymorphic sites in 61 zucchini materials with an average of 4.18 loci per pair of primers (Additional file 2, Table S11). The number of observed alleles (Na) ranged from 2 to 9. The highest number of $\mathrm{Na}$ (9) were observed by SSR010246, SSR026560, SSR026918, SSR027656, and SSR026980, followed by SSR011546, SSR003315 and SSR026797 
with eight alleles. The number of effective alleles $(\mathrm{Ne})$ varied from 1.03 to 6.07 with an average of 2.31. The Shannon information index (I) ranged from 0.083 to 1.96 with an average of 0.83 . The PIC value ranged from 0.03 to 0.83 with an average of 0.43 .

According to the results of the structure operation, it could be seen that when $K=2$, $\Delta \mathrm{K}$ showed a significant peak value, indicating that 61 materials selected in this study could be divided into two groups (Additional file 1, Fig S2), named group I and group II. The group I contained 5 materials $(8.20 \%)$, all of them were wild varieties; The group II contained 56 materials (91.80\%), which were all cultivars (Fig. 5A). It indicated that our primers could clearly distinguish the cultivated materials from the wild varieties, and the backgrounds of the choosed materials were narrow. Furthermore, the phylogenetic tree was drawn by MEGA6.0 software (Fig. 5B), the 5 materials $(2,29,30,31$ and 45$)$ in group I were at the bottom of the phylogenetic tree, indicating that, based on the genetic analysis, the 61 zucchini materials were also divided into two clusters, which were completely in accordance with the result of structure analysis.

\section{Discussion}

\section{Frequency, distribution and characterization of microsatellite in three Cucurbita genomes}

With the development of the sequencing technology, the discovery and mining of genomic SSR loci had successful applications in many plant species such as cotton [35, 36], foxtail millet [37], cucumber [38], watermelon [39], tobacco [40] and melon [23]. Nevertheless, little effort has been paid on Cucurbita species. In this study, the density of the SSR markers in the genome of the Cucurbita species was about 113$145 \mathrm{SSR} / \mathrm{Mb}$, C. pepo had the minimum length of the genome size and maximum number of microsatellites. The number of microsatellites and their density identified in this study was lower than that in cucumber (552 SSR/Mb), but was higher than that in melon (109 SSR/Mb) and watermelon (111 SSR/Mb) [23, 38, 39]. Except the natural difference among different genomes, there are many factors that can affect the deviations of the SSR density, different software, different parameter settings, and sequencing depths. For example, in this paper, basic motifs from dinucleotides to octanucleotides were checked with a minimum length of 18 bp (for di- and 
tetranucleotides), $20 \mathrm{bp}$ (for pentanucleotides), $24 \mathrm{bp}$ (for hexanucleotides), $21 \mathrm{bp}$ (for heptanucleotides), and 24 bp (for octanucleotides). In cucumber [38], though they also checked the motifs from dinucleotides to octanucleotides, their minimum lengths (12 bp for di- and tetranucleotides, $15 \mathrm{bp}$ for pentanucleotides, $18 \mathrm{bp}$ for hexanucleotides, $21 \mathrm{bp}$ for heptanucleotides, and $24 \mathrm{bp}$ for octanucleotides) were mostly shorter than our setting. The size of assembled grass genomes was higher than that in Cucurbita species, which leading to their higher SSR numbers than ours.

In this research, we analyzed the distribution and frequency of microsatellites in assembled Cucurbita genome. 36,234 polymorphic SSR markers in C. pepo identified by in silico analysis showed that $40 \%(14,489)$ and $18 \%(6,578)$ of them were dinucleotides and trinucleotides types, respectively (Table. 1). Previous studies have shown that the dinucleotides motifs with high repeat numbers were more polymorphic compared to those with short repeat units [41]. Consistent with the studies in watermelon and melon, the frequency analysis of various nucleotide repeat types in Cucurbita genus indicated that dinucleotide repeats were the most popular SSRs, followed by tri-, tetra-, penta-, hepta-, hexa- and octonucleotide repeats [23, 39]. However, this was different with the studies in other species. For example, the density of tetranucleotide repeats were the most abundant in Cucumis sativus (164.2 SSR/Mb), Populus trichocarpa (144.9 SSR/Mb), Medicago truncatula (102.8 SSR/Mb), and Vitis vinifera (171.3 SSR/Mb), and the trinucleotide repeats were the most abundant in Arabidopsis thaliana (146.6 SSR/Mb), Glycine max (103.1 SSR/Mb), and Oryza sativa (220.1 SSR/Mb) [38]. These results indicated that the most popular SSR motifs were varied in different species.

A general negative correlation was observed between the microsatellite frequency and the number of repeat units. Furthermore, AT or AAT type prevailed in the dicot plants [20]. Our results agreed with this result. Lately, SSR markers analysis in bitter gourd showed that all kinds of triple repeat units were the main type. A/T, AT/AT, AAT/ATT, and AAAT/ATTT were overrepresented and totally accounted for 63.86$76.30 \%$ of all motifs identified in the seven cucurbit genomes, respectively [42]. This pattern was also found in other genomes [18, 43, 44]. On the contrary, the frequency of the GC or CCG type was very low in the genomic level $[45,46]$. GC, TC or GA 
types have relatively stable structures. Most of the AT types distribute in non-genic regions and TC/GA types in coding sequences [40]. It has been reported that many bacteria SSRs in intergenic regions have regulating functions [47]. Whether these pumpkin SSR motifs played any role during specialization or protein coding was uncertain.

\section{The chromosome synteny analysis between $C$. pepo and other Cucurbitaceae species by cross-species transferable markers}

The chromosome synteny analysis has been carried out in many plants, such as cucumber, watermelon and melon [23], but little has been reported in Cucurbita genus. In this study, a large number of cross-species SSR markers were developed in Cucurbita genome, and they enabled us to investigate the syntenic blocks at a high resolution. Though the size of the pumpkin genome is similar to that of the other sequenced Cucurbitaceae species, the number of cross-species SSR markers in Cucurbita genus is much higher. Compared to hundreds of shared markers in previous studies [21], much more cross-species transferable SSR markers in Cucurbita genus were used for chromosome synteny analysis in our study. It might be the case that there had a WGD event leading to the high abundance of SSR markers [8, 9], but it's not observed in other sequenced Cucurbitaceae species, such as cucumber [15], melon [48], watermelon [16].

After the genome comparison between C. pepo and cucumber, C. pepo and melon, C. pepo and watermelon, 52, 61 and 89 syntenic blocks were identified, respectively. These blocks distributed on all chromosomes. The similarly works among Cucurbita species was also reported in previous study, about $63.2 \%, 58.7 \%$, and $68.3 \%$ of the $C$. maxima genomic regions were syntenic to melon, cucumber, and watermelon, respectively, while those numbers changed to $64.0 \%, 62.2 \%$, and $69.5 \%$ in $C$. moschata, and they were composed of homoeologous blocks [9]. In most cases, because of the chromosome fission, the multiple synteny relationships between $C$. pepo and other Cucurbitaceae species were shown. The most complicated syntenic pattern existed in Chr1 of $C$. pepo, which was syntenic to seven watermelon chromosomes, indicating that complex structure changes occurred after their divergence from the ancestor. The ratio of collinear-blocks to inversion-blocks was 
nearly 1:1 in Cucurbita genus, the possible reason was that the genome duplication and interchromosomal exchanges happened randomly [9].

According to the cross-species SSR markers analysis, we identified more syntenic blocks in Cucurbita species than that in melon, cucumber or watermelon. At the same time, we found that each block contained abundant SSR markers. For example, the $C$. pepo syntenic block CpeCmos53 had the largest number of shared SSR markers $(1,080)$. As we all known, the largest number of shared SSR markers (386) between melon and cucumber was found in block C3 [23]. This indicated that there were lots of genetic variations and highly evolutionary relationships among homologous species. The single chromosomal analysis by cross-species shared SSR markers showed that there were six large-scale inversion regions in different chromosomes between $C$. pepo and $C$. moschata or between $C$. pepo and $C$. maxima. The previous study reported that all chromosomes except Chr4 contained only homologous blocks between $C$. maxima and $C$. moschata [9]. This might be due to genome duplication, large-scale inter-chromosomal exchanges or long-term evolutionary forces. It also indicated that $C$. pepo had more complex evolutionary processes than other Cucurbita species.

\section{The genetic diversity and population structure of $C$. pepo}

As one of the species with the most complex genetic background, limited molecular markers have been used for genetic diversity and population structure analysis in $C$. pepo. For example, Ferriol et al. used SRAP and AFLP markers to analyze the population structure in 69 C. pepo species, and found that SRAP markers were more consistent with the morphological character [49]. Nonthuko et al. used nine RAPD markers and ten SSR primers to study genetic diversity in seven $C$. pepo inbred lines and detected 100 and 56 alleles, respectively, but only the RAPD markers could distinguish these cultivars according to their fruit colors [50]. Paris et al. used AFLP, ISSR and SSR markers to analyze the relationship between wild and domestic species, and successfully clustered them into three groups based on their fruit colors, fruit sizes and origins [26]. Because of the scarcity of highly polymorphic markers and genome-sequencing data, most previous studies in Cucurbita species have been done using low throughput and anonymous markers. In this study, we developed 91,248 
SSR markers from the three Cucurbita genomes, they would benefit the genetic studies in the future.

Genome wide SSR markers were not developed until the completion of the genomic sequencing in Cucurbita species. In this study, we employed 66 pairs of high polymorphic SSR markers to investigate the genetic diversity of 61 C. pepo materials and an average of 4.18 alleles were amplified. The UPGMA cluster analysis results were almost consistent with the results of population structure analysis (Fig. 5). In this study, we have successfully clustered these materials into two subspecies, subsp. Ovifer (or subsp.texana) and subsp. pepo. Most of C. pepo subsp. pepo accessions were clustered into group II, and the five C. pepo subsp. Ovifer accessions were clustered into group I. This result was consistent with the previous studies [29, 49], but Decker classificated C. pepo into three subspecies, C. pepo subsp. fraterna (Bailey) Andres, C. pepo subsp.texana (Scheele) Filov and C. pepo subsp. Pepo [51]. C. pepo subsp. fraterna has been considered as the putative ancestor for the species as a whole and consists exclusively of wild gourds from northeastern Mexico [52]. Moreover, the genetic structure revealed that the background of some materials was a mixed model between group I and group II. For example, the material 45 in group I has complex genetic background, just like the material 14 and 16 in group II, indicating that these accessions may have undergone gene exchange between two subspecies.

The Cucurbita species is an economically important crop, but its breeding process fall behind the other Cucurbitaceous species. Limited high-quality cultivars cannot meet the production requirements. Thus, speeding the current breeding programs using MAS become more and more important. The whole-genome SSR markers detected in this study will promote the development and the utilization in basic and applied research.

\section{Conclusions}

In this paper, based on reference sequences of three Cucurbita species, a total of 91,248 SSR markers were developed at the whole genome level. Then their frequency and distribution were detected and analysed. According to these markers, the cross- 
species SSR markers were checked by silico-PCR and the synteny relationships were analysed between C.pepo and other Cucurbitaceae species. Furthermore, 66 polymorphism SSR markers were employed to check the genetic diversity of C.pepo. These SSR markers will benefit the related researches and accelerate the process of pumpkin breeding.

\section{Methods}

\section{Plant Materials}

All the pumpkin materials used in this study were introduced from the national crop germplasm resource platform of china (sub-platform of vegetable germplasm resource) in 2018, among which 4 materials came from Russia, 1 from America and 56 materials came from 17 provinces of China. The number and sources were shown in Table S1.

\section{Genome SSR identification and development in Cucurbitaceae}

The genome information of watermelon, melon, cucumber and pumpkin was downloaded from http://cucurbitgenomics.org/. To develop a set of higher polymorphism SSR primers for the future study, the criteria used for microsatellite identification in this study was from 2 to $8 \mathrm{bp}$, and mononucleotides were not considered due to the difficulty in distinguishing bona fide microsatellites from sequencing or assembly error. The Microsatellite Identification tool (MISA) was used to identify and analyze SSR markers, including perfect and compound microsatellites. Specific screening details were as follows: repeats with a minimum length of $18 \mathrm{bp}$ (for di- and tetranucleotides), $20 \mathrm{bp}$ (for pentanucleotides), $24 \mathrm{bp}$ (for hexanucleotides), $21 \mathrm{bp}$ (for heptanucleotides), and $24 \mathrm{bp}$ (for octanucleotides). The oligonucleotide primers for these SSRs were designed according to the flanking genomic sequence using Primer3 software (v.1.1.4). Primers were designed to generate amplicons of 100-300 bp in length with the following minimum, optimum and maximum values for Primer3 parameters: primer length (bp): 18-20-24; Tm $\left({ }^{\circ} \mathrm{C}\right)$ : 50-55-60. Other parameters used the default program values.

\section{In silico PCR and synteny analysis of SSR markers in Cucurbitaceae}


Using the zucchini (C. pepo MU-CU-16) genome SSR markers as a reference, we comparatively analyzed the genome SSR information of cucumber (Gy14), melon (DH92), watermelon (97103), C. moschata cv. Rifu and C. maxima cv. Rimu, respectively. This was performed with a custom Perl script that used the NCBI BLASTN program as a search engine with expect value of 10 and filtering. We allowed up to five nucleotide mismatches at the 5 'end of the primer but no mismatches at the 3 'end, and a minimum of $90 \%$ overall match homology. To establish the syntenic relationships of chromosomes between C.pepo and C. sativus, $C$. lanatus, C. melo, C. maxima or C.moschata. We discarded the repeat SSR markers in different genomes, only kept the SSR markers in these genomes, which had single silico-PCR product. Besides, these shared SSR markers located on the chromosomal unanchored scaffolds were further filtered. The SSR marker-based syntenic relationships were finally visualized with visualization blocks in Circos software v0.55 [53].

\section{Genomic DNA extraction, PCR amplification and electrophoresis detection}

Genomic DNA of all the materials was extracted using $1 \mathrm{~g}$ young leaf sample with the CTAB method. The extracted DNA was dissolved in 1×TE buffer. The concentration and purity were detected by Nanodrop-2000 nucleic acid analyzer. The extracted DNA was diluted to $30 \mathrm{ng} / \mu \mathrm{l}$ as a working solution, and kept at $4{ }^{\circ} \mathrm{C}$.

Each PCR reaction contained $1 \mu \mathrm{l}$ of template DNA, $0.5 \mu \mathrm{l}$ each of forward and

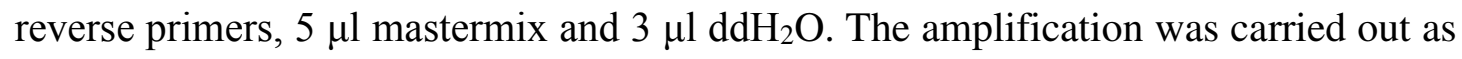
follows: an initial denaturing step at $95{ }^{\circ} \mathrm{C}$ for $5 \mathrm{~min}, 94{ }^{\circ} \mathrm{C}$ for $30 \mathrm{~s}$, followed by 6 cycles of $68-58{ }^{\circ} \mathrm{C}$ for $45 \mathrm{~s}$, each cycle was reduced by $2{ }^{\circ} \mathrm{C}$, each annealing time was $1 \mathrm{~min}$, and $72{ }^{\circ} \mathrm{C}$ for $1 \mathrm{~min} ; 30$ cycles of $94{ }^{\circ} \mathrm{C}$ for $30 \mathrm{~s}, 50{ }^{\circ} \mathrm{C}$ for $30 \mathrm{~s}$, and $72{ }^{\circ} \mathrm{C}$ for 1 min; In the last cycle, primer extension was performed at $72{ }^{\circ} \mathrm{C}$ for $10 \mathrm{~min}$.

PCR products were analyzed by $9 \%$ polyacrylamide gel electrophoresis, and $100 \mathrm{bp}$ DNA ladder was used as the reference marker. After electrophoresis, silver staining was performed to display the PCR products, and photos were taken for preservation.

\section{Cluster calculating}

The heterozygosity (He), observer gene number $(\mathrm{Na})$, effective alleles $(\mathrm{Ne})$, observed heterozygosity (Ho) and Shannon-Weaver index (I) were calculated by the pop-gen 
software. Polymorphism information content (PIC) of SSR markers was computed by EXCEL. When the PIC of a SSR marker was below 0.25, it was considered as a low polymorphism marker, and it was considered as a high polymorphism marker if its PIC was above 0.5.

These amplification bands of each SSR-PCR primer pairs were separated by polyacrylamide gel electrophoresis. The number of alleles stood by the total number of these bands in this SSR loci. In the same location, having band was marked as "1", no band was marked as "0", and the missing band was marked as "-1". In this paper, we used Genalex-6 software to conduct the matrix calculation of SSR marker data which had been assigned a value, then transformed it into triangle matrix, saved it as a mega-file, finally, imported the mega-file into the Mega-6.0 software, and selected UPGMA algorithm in "phylogeny" drop-down menu to draw the cluster diagram [54].

The software Structure V2.3 was used to analyze the population structure [55, 56]. An admixture model and correlated allele frequencies was used to estimate the number of the populations. For each of the K-values (range from 1 to 5), ten independent runs were performed with a burn-in period of 100,000 followed by 500,000 Markov Chain Monte Carlo. The optimal K-values depends on the peak of $\mathrm{K}=$ mean $(|\operatorname{Ln} " \mathrm{P}(\mathrm{D})|) /(\operatorname{sdLnP}(\mathrm{D}))$. Based on the structure results, the most probable $\mathrm{K}$ value was analysis by the Structure Harvester (http://taylorO.biology.ucla.edu/ struct_harvest/).

\section{List of abbreviations}

SSR, simple sequence repeats; QTL, quantitative trait loci; AFLP, amplified fragment length polymorphism; RAPD, random amplified polymorphic DNA; SNP, single nucleotide polymorphism; WGD, whole genome duplication; PCR, polymerase chain reaction; ISSR, Inter-simple sequence repeat: UPGMA, unweighted Pair Group Method with Arithmetic Mean.

\section{Declarations}

\section{Ethics approval and consent to participate}


Not applicable

\section{Consent for publication}

Not applicable

\section{Availability of data and materials}

All data generated or analysed during this study are reflected in the present article. Our datasets of materials and genome-wide SSR markers were listed in Additional file 2.

\section{Competing interests}

The authors declare that they have no competing interests.

\section{Funding}

This research was supported by Science and Technology Project of Henan Province (agriculture) (182102110280) and Innovation and Entrepreneurship Training Program for College Students (201910466017), China.

\section{Authors' contributions}

LZ and HYZ performed the data analysis and wrote the manuscript. YML and XBW conducted validation of SSR polymorphism, genetic diversity and population structure analysis. JTL, ZLZ and YJW participated in SSR genetic diversity analysis. JBH, SY and YQL involved in the germplasm collection and growth. SSR and LMY designed this paper and revised the manuscript. All authors read and approved the final manuscript.

\section{Acknowledgements}

The authors are highly grateful to Professor Yufeng Wu of Nanjing Agricultural University in Circos analysis. The C.pepo materials used in this study were introduced from the national crop germplasm resource platform of china. Our thanks for the help of Jiangping Song from Chinese Academy of Agricultural Sciences in material's 
introduction. We are also indebted to the editor and reviewers for critically evaluating the manuscript and providing constructive comments for its improvement.

\section{Reference:}

1. Toxic plants of North America. Choice: Current Reviews for Academic Libraries 2013, 50(12):22022203.

2. Loy JB: Morpho-physiological aspects of productivity and quality in squash and pumpkins (Culcurbita spp.). Crit Rev Plant Sci 2004, 23(4):337-363.

3. Nee M: The domestication of cucurbita (Cucurbitaceae). Econ Bot 1990, 44(3):56-68.

4. Smith BD: The Initial Domestication of Cucurbita pepo in the Americas 10,000 Years Ago. Science 1997, 276(5314):932-934.

5. Ferriol M, Picó B: Pumpkin and Winter Squash. In: Vegetables I: Asteraceae, Brassicaceae, Chenopodicaceae, and Cucurbitaceae. Edited by Prohens J, Nuez F. New York, NY: Springer New York; 2008: 317-349.

6. Kates HR, Soltis PS, Soltis DE: Evolutionary and domestication history of Cucurbita (pumpkin and squash) species inferred from 44 nuclear loci. Mol Phylogenet Evol 2017, 111:98-109.

7. Arumuganathan K, Earle ED: Nuclear DNA content of some important plant species. Plant Molecular Biology Reporter 1991, 9(3):208-218.

8. Montero-Pau J, Blanca J, Bombarely A, Ziarsolo P, Esteras C, Marti-Gomez C, Ferriol M, Gomez P, Jamilena M, Mueller L et al: De novo assembly of the zucchini genome reveals a whole-genome duplication associated with the origin of the Cucurbita genus. Plant Biotechnol J 2018, 16(6):11611171 .

9. Sun HH, Wu S, Zhang GY, Jiao C, Guo SG, Ren Y, Zhang J, Zhang HY, Gong GY, Jia ZC et al: Karyotype Stability and Unbiased Fractionation in the Paleo-Allotetraploid Cucurbita Genomes. Mol Plant 2017, 10(10):1293-1306.

10. Savage JA, Haines DF, Holbrook NM: The making of giant pumpkins: how selective breeding changed the phloem of Cucurbita maxima from source to sink. Plant Cell Environ 2015, 38(8):15431554 .

11. Davis AR, Perkins-Veazie P, Sakata Y, Lopez-Galarza S, Maroto JV, Lee SG, Huh YC, Sun ZY, Miguel A, King SR et al: Cucurbit grafting. Crit Rev Plant Sci 2008, 27(1):50-74.

12. Lee JM, Kubota C, Tsao SJ, Bie Z, Echevarria PH, Morra L, Oda M: Current status of vegetable grafting: Diffusion, grafting techniques, automation. Sci Hortic-Amsterdam 2010, 127(2):93-105.

13. Lv J, Qi JJ, Shi QX, Shen D, Zhang SP, Shao GJ, Li H, Sun ZY, Weng YQ, Shang Y et al: Genetic Diversity and Population Structure of Cucumber (Cucumis sativus L.). Plos One 2012, 7(10). 
14. Li Y, Wen C, Weng Y: Fine mapping of the pleiotropic locus B for black spine and orange mature fruit color in cucumber identifies a $50 \mathrm{~kb}$ region containing a R2R3-MYB transcription factor. Theor Appl Genet 2013, 126(8):2187-2196.

15. Huang SW, Li RQ, Zhang ZH, Li L, Gu XF, Fan W, Lucas WJ, Wang XW, Xie BY, Ni PX et al: The genome of the cucumber, Cucumis sativus L. Nat Genet 2009, 41(12):1275-U1229.

16. Guo SG, Zhang JG, Sun HH, Salse J, Lucas WJ, Zhang HY, Zheng Y, Mao LY, Ren Y, Wang ZW et al: The draft genome of watermelon (Citrullus lanatus) and resequencing of 20 diverse accessions. Nat Genet 2013, 45(1):51-+.

17. Jordi Garcia-Mas AB, Walter Sanseverino, Michael Bourgeois, Gisela Mir, Víctor M. González, Elizabeth Hénaff, Francisco Câmara, Luca Cozzuto, Ernesto Lowy, Tyler Alioto, Salvador CapellaGutiérrez, Jose Blanca, Joaquín Cañizares, Pello Ziarsolo, Daniel Gonzalez-Ibeas, Luis RodríguezMoreno, Marcus Droege, Lei Du, Miguel Alvarez-Tejado, Belen Lorente-Galdos, Marta Melé, Luming Yang, Yiqun Weng, Arcadi Navarro, Tomas Marques-Bonet, Miguel A. Aranda, Fernando Nuez, Belén Picó, Toni Gabaldón, Guglielmo Roma, Roderic Guigó, Josep M. Casacuberta, Pere Arús, and Pere Puigdomènech The genome of melon (Cucumis melo L.). Proceedings of the National Academy of Sciences 2012.

18. Cavagnaro PF, Senalik DA, Yang L, Simon PW, Harkins TT, Kodira CD, Huang S, Weng Y: Genomewide characterization of simple sequence repeats in cucumber (Cucumis sativus L.). BMC Genomics 2010, 11:569.

19. Zhu H, Guo L, Song P, Luan F, Hu J, Sun X, Yang L: Development of genome-wide SSR markers in melon with their cross-species transferability analysis and utilization in genetic diversity study. Mol Breeding 2016, 36(11):153.

20. Zhu H, Song P, Koo DH, Guo L, Li Y, Sun S, Weng Y, Yang L: Genome wide characterization of simple sequence repeats in watermelon genome and their application in comparative mapping and genetic diversity analysis. BMC Genomics 2016, 17:557.

21. Li DW, Cuevas HE, Yang LM, Li YH, Garcia-Mas J, Zalapa J, Staub JE, Luan FS, Reddy U, He XM et al: Syntenic relationships between cucumber (Cucumis sativus L.) and melon (C. melo L.) chromosomes as revealed by comparative genetic mapping. Bmc Genomics 2011, 12.

22. Yang LM, Koo DH, Li DW, Zhang T, Jiang JM, Luan FS, Renner SS, Henaff E, Sanseverino W, GarciaMas $\mathrm{J}$ et al: Next-generation sequencing, FISH mapping and synteny-based modeling reveal mechanisms of decreasing dysploidy in Cucumis. Plant $J$ 2014, 77(1):16-30.

23. Zhu HY, Guo LQ, Song PY, Luan FS, Hu JB, Sun XF, Yang LM: Development of genome-wide SSR markers in melon with their cross-species transferability analysis and utilization in genetic diversity study. Mol Breeding 2016, 36(11).

24. Hi LY, Jeong JH, Hong KH, Dong KB: Use of Random Amplified Polymorphic DNAs for Linkage Group Analysis in Interspecific Hybrid $\mathbf{F}_{2}$ Generation of Cucurbita. Horticulture Environment \& Biotechnology 1995, 36(3):323-330. 
25. Brown RN, Myers JR: A genetic map of squash (Cucurbita sp.) with randomly amplified polymorphic DNA markers and morphological markers. Journal of the American Society for Horticulturalence 2002, 127(4):568-575.

Paris HS, Yonash N, Portnoy V, Mozes-Daube N, Tzuri G, Katzir N: Assessment of genetic relationships in Cucurbita pepo (Cucurbitaceae) using DNA markers. Theor Appl Genet 2003, 106(6):971-978.

27. Zraidi A, Stift G, Pachner M, Shojaeiyan A, Gong L, Lelley T: A consensus map for Cucurbita pepo. Mol Breeding 2007, 20(4):375-388.

28. Gong L, Stift G, Kofler R, Pachner M, Lelley T: Microsatellites for the genus Cucurbita and an SSRbased genetic linkage map of Cucurbita pepo L. Theoretical and Applied Genetics 2008, 117(1):37-48. Esteras C, Gomez P, Monforte AJ, Blanca J, Vicente-Dolera N, Roig C, Nuez F, Pico B: Highthroughput SNP genotyping in Cucurbita pepo for map construction and quantitative trait loci mapping. Bmc Genomics 2012, 13.

30. Blanca J, Canizares J, Roig C, Ziarsolo P, Nuez F, Pico B: Transcriptome characterization and high throughput SSRs and SNPs discovery in Cucurbita pepo (Cucurbitaceae). Bmc Genomics 2011, 12.

31. Wyatt LE, Strickler SR, Mueller LA, Mazourek M: An acorn squash (Cucurbita pepo ssp. ovifera) fruit and seed transcriptome as a resource for the study of fruit traits in Cucurbita. Hortic ResEngland 2015, 2.

32. Xanthopoulou A, Psomopoulos F, Ganopoulos I, Manioudaki M, Tsaftaris A, Nianiou-Obeidat I, Madesis P: De novo transcriptome assembly of two contrasting pumpkin cultivars. Genom Data 2016, 7:200-201.

33. Aliki X, Ganopoulos I, Psomopoulos F, Manioudaki M, Moysiadis T, Kapazoglou A, Osathanunkul M, Michailidou S, Kalivas A, Tsaftaris A et al: De novo comparative transcriptome analysis of genes involved in fruit morphology of pumpkin cultivars with extreme size difference and development of EST-SSR markers, vol. 622; 2017.

34. Vitiello Alessia SD, D’Agostino Nunzio, Digilio Maria Cristina, Pennacchio, Francesco CG, Rao Rosa: Unraveling zucchini transcriptome response to aphids. Peer J PrePrints 2016, 4.

35. Lu CR, Zou CS, Zhang YP, Yu DQ, Cheng HL, Jiang PF, Yang WC, Wang QL, Feng XX, Prosper MA et al: Development of chromosome-specific markers with high polymorphism for allotetraploid cotton based on genome-wide characterization of simple sequence repeats in diploid cottons (Gossypium arboreum L. and Gossypium raimondii Ulbrich). Bmc Genomics 2015, 16.

36. Wang Q, Fang L, Chen JD, Hu Y, Si ZF, Wang S, Chang LJ, Guo WZ, Zhang TZ: Genome-Wide Mining, Characterization, and Development of Microsatellite Markers in Gossypium Species. Sci Rep-Uk 2015, 5.

37. Zhang S, Tang CJ, Zhao Q, Li J, Yang LF, Qie LF, Fan XK, Li L, Zhang N, Zhao MC et al: Development of highly polymorphic simple sequence repeat markers using genome-wide microsatellite variant analysis in Foxtail millet [Setaria italica (L.) P. Beauv.]. Bmc Genomics 2014, 15. 
38. Cavagnaro PF, Senalik DA, Yang LM, Simon PW, Harkins TT, Kodira CD, Huang SW, Weng YQ: Genome-wide characterization of simple sequence repeats in cucumber (Cucumis sativus $\mathbf{L}$.). Bmc Genomics 2010, 11.

39. Zhu HY, Song PY, Koo DH, Guo LQ, Li YM, Sun SR, Weng YQ, Yang LM: Genome wide characterization of simple sequence repeats in watermelon genome and their application in comparative mapping and genetic diversity analysis. Bmc Genomics 2016, 17.

40. Wang XW, Yang S, Chen YD, Zhang SM, Zhao QS, Li M, Gao YL, Yang L, Bennetzen JL: Comparative genome-wide characterization leading to simple sequence repeat marker development for Nicotiana. Bmc Genomics 2018, 19.

41. Weber JL: Informativeness of human (dC-dA)n.(dG-dT)n polymorphisms. Genomics 1990, 7(4):524-530.

42. Cui J, Cheng J, Nong D, Peng J, Hu Y, He W, Zhou Q, Dhillon NPS, Hu K: Genome-Wide Analysis of Simple Sequence Repeats in Bitter Gourd (Momordica charantia). Front Plant Sci 2017, 8.

43. Kim TS, Booth JG, Gauch HG, Jr., Sun Q, Park J, Lee YH, Lee K: Simple sequence repeats in Neurospora crassa: distribution, polymorphism and evolutionary inference. Bmc Genomics 2008, 9:31.

44. Cheng J, Zhao Z, Li B, Qin C, Wu Z, Trejo-Saavedra DL, Luo X, Cui J, Rivera-Bustamante RF, Li S et al: A comprehensive characterization of simple sequence repeats in pepper genomes provides valuable resources for marker development in Capsicum. Sci Rep 2016, 6:18919.

45. Tangphatsornruang S, Somta P, Uthaipaisanwong P, Chanprasert J, Sangsrakru D, Seehalak W, Sommanas W, Tragoonrung S, Srinives P: Characterization of microsatellites and gene contents from genome shotgun sequences of mungbean (Vigna radiata (L.) Wilczek). Bmc Plant Biol 2009, 9:137.

46. Portis E, Lanteri S, Barchi L, Portis F, Valente L, Toppino L, Rotino GL, Acquadro A: Comprehensive Characterization of Simple Sequence Repeats in Eggplant (Solanum melongena L.) Genome and Construction of a Web Resource. Front Plant Sci 2018, 9.

47. Zhao ZX, Guo C, Sutharzan S, Li P, Echt CS, Zhang J, Liang C: Genome-Wide Analysis of Tandem Repeats in Plants and Green Algae. G3-Genes Genom Genet 2014, 4(1):67-78.

48. Garcia-Mas J, Benjak A, Sanseverino W, Bourgeois M, Mir G, Gonzalez VM, Henaff E, Camara F, Cozzuto L, Lowy E et al: The genome of melon (Cucumis melo L.). P Natl Acad Sci USA 2012, 109(29):11872-11877.

49. Ferriol M, Pico B, Nuez F: Genetic diversity of a germplasm collection of Cucurbita pepo using SRAP and AFLP markers. Theoretical And Applied Genetics 2003, 107(2):271-282.

50. Ntuli NR, Tongoona PB, Zobolo AM: Genetic diversity in Cucurbita pepo landraces revealed by RAPD and SSR markers. Sci Hortic-Amsterdam 2015, 189:192-200.

51. Decker DS: Origin (s), Evolution, and Systematics of Cucurbita pepo (Cucurbitaceae). Econ Bot 1988, 42:4-15. 
52. Paris HS: Germplasm enhancement of Cucurbita pepo (pumpkin, squash, gourd: Cucurbitaceae): progress and challenges. Euphytica 2015, 208(3):415-438.

53. Krzywinski M, Schein J, Birol I, Connors J, Gascoyne R, Horsman D, Jones SJ, Marra MA: Circos: an information aesthetic for comparative genomics. Genome Res 2009, 19(9):1639-1645.

54. Peakall R, Smouse PE: GenAlEx 6.5: genetic analysis in Excel. Population genetic software for teaching and research-an update. Bioinformatics 2012, 28(19):2537-2539.

55. Pritchard JK, Stephens M, Donnelly P: Inference of population structure using multilocus genotype data. Genetics 2000, 155(2):945-959.

56. Evanno G, Regnaut S, Goudet J: Detecting the number of clusters of individuals using the software STRUCTURE: a simulation study. Mol Ecol 2005, 14(8):2611-2620. 
Figures

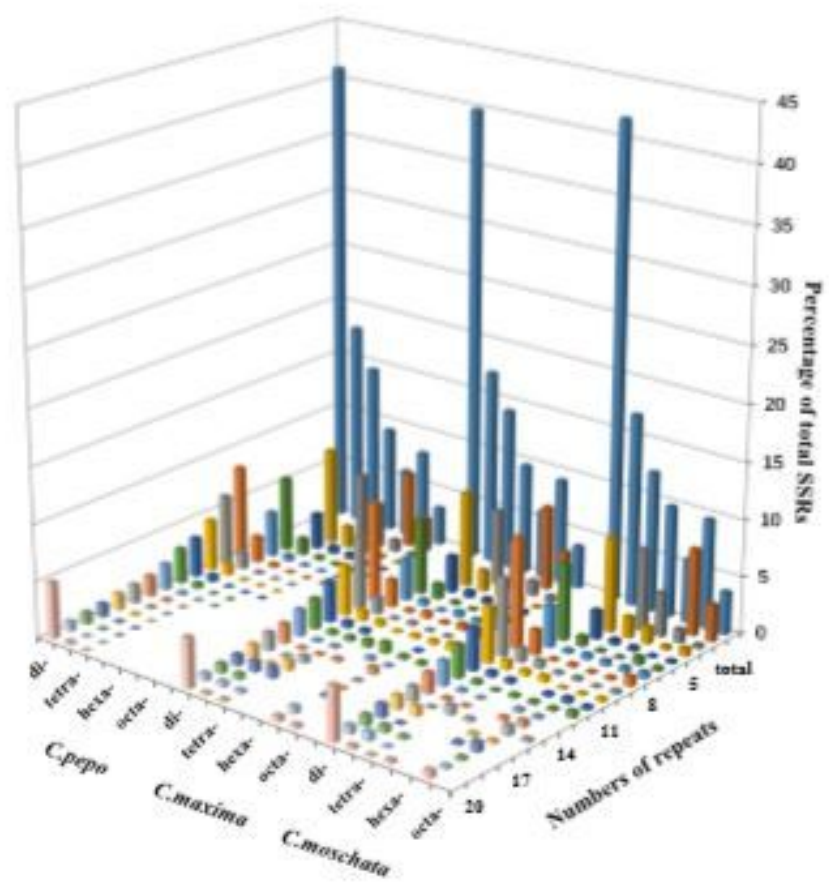

\section{Figure 1}

Distribution of SSR motif repeat numbers and relative frequency in Cucurbita genome. The vertical axis shows the abundance of microsatellites that have different motif repeat numbers (from 3 to $>20$ ) with different colors. 


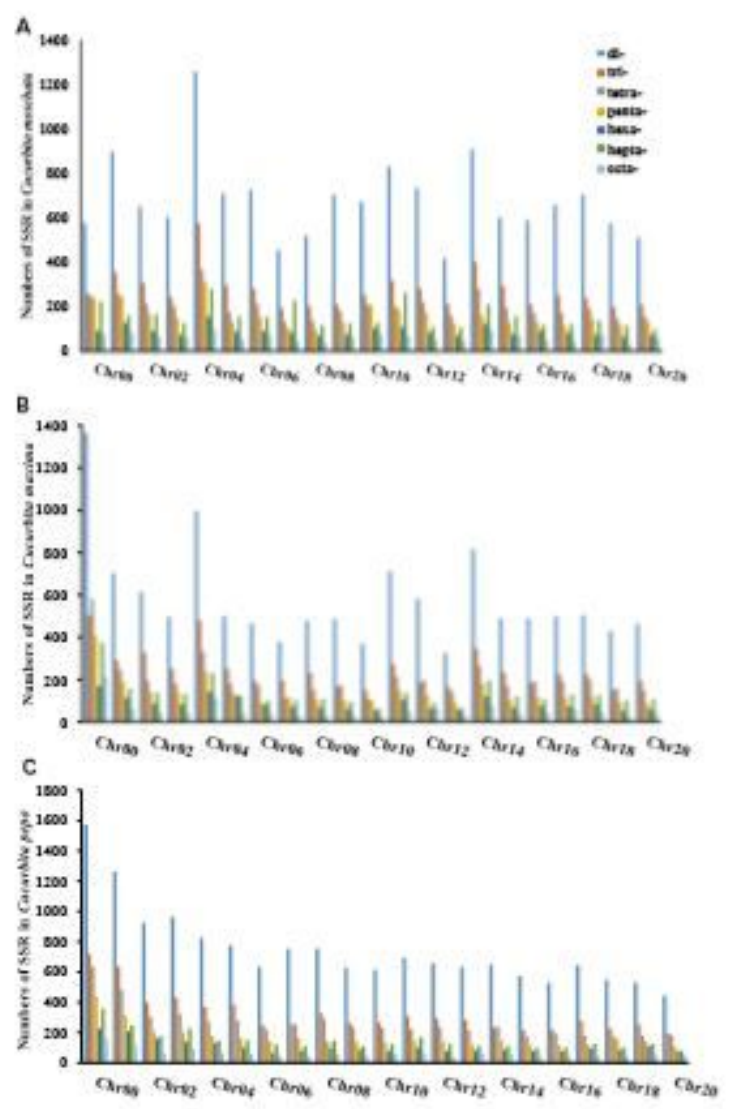

\section{Figure 2}

The distribution of SSR repeat types on each chromosome in C.moschata. The vertical axis shows the number of microsatellites from di-nucleotide to octo-nucleotide which are discriminated by different colors. The horizontal axis show different chromosomes of C.moschata and chr0 means all the chromosome unanchored scaffolds. B, The distribution of SSR repeat types on each chromosome in C.maxima. The vertical axis shows the number of microsatellites from di-nucleotide to octo-nucleotide which are discriminated by different colors. The horizontal axis show different chromosomes of C.maxima and chr0 means all the chromosome unanchored scaffolds. C, The distribution of SSR repeat types on each chromosome in C.pepo. The vertical axis shows the number of microsatellites from dinucleotide to octo-nucleotide which are discriminated by different colors. The horizontal axis show different chromosomes of C.pepo and LG00 means all the chromosome unanchored scaffolds. 
A

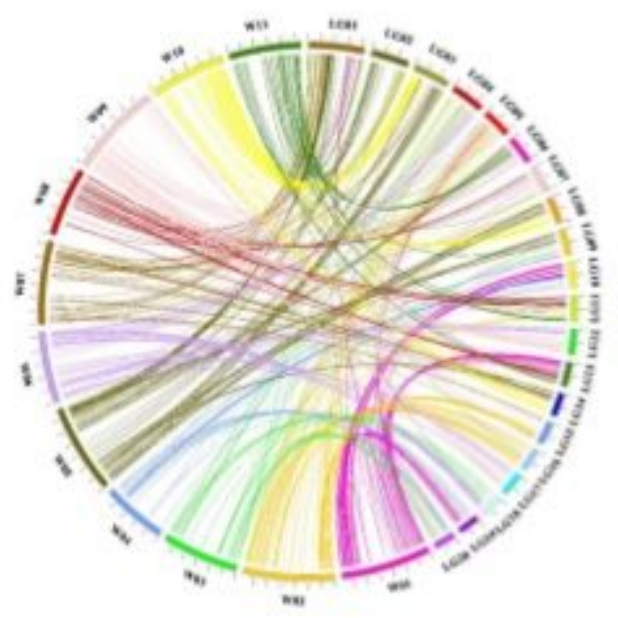

B

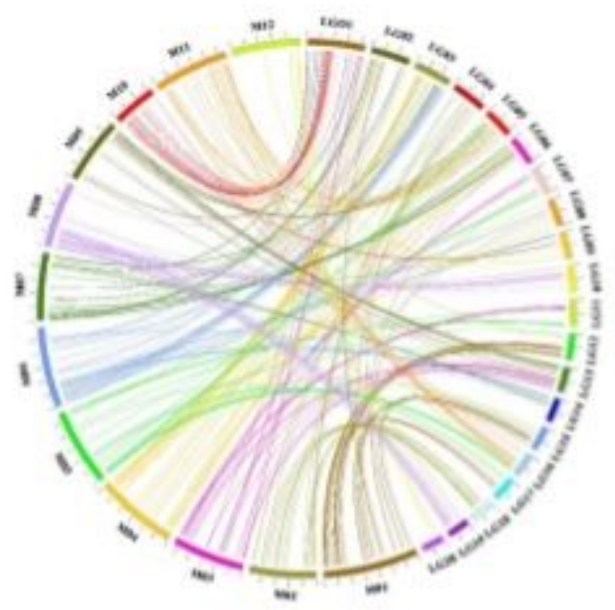

C

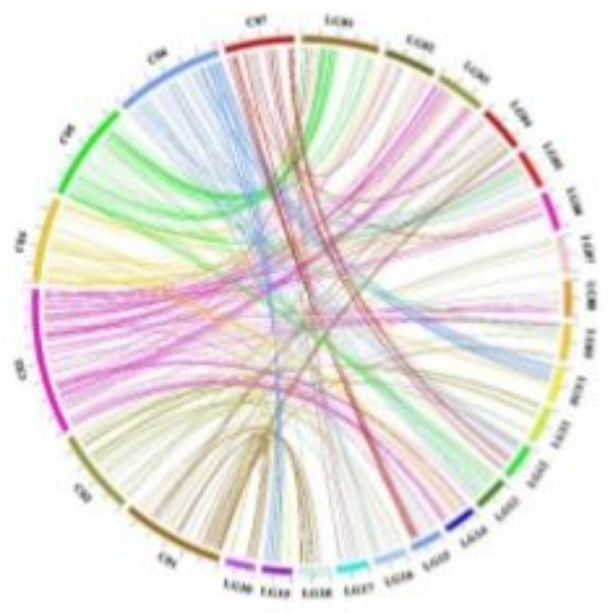

Figure 3

Syntenic relationships of C.pepo with C. lanatus (A), C. melo (B) and C. sativus (C). Chromosome synteny between C.pepo and C.sativus is based on 391 cross-species markers; synteny between C.pepo and C.melo is based on 425 cross-species markers; synteny between C.pepo and C.lanatus is based on 717 cross-species markers. W1 W11 represent C.Ianatus eleven chromosomes, M01-M12 represent C.melo twelve chromosomes, C01-C07 represent C.sativus seven chromosomes, and LG01-LG20 represent C.prpo 
twenty chromosomes. Syntenic blocks are connected by with the same color lines from C.pepo chromosomes.
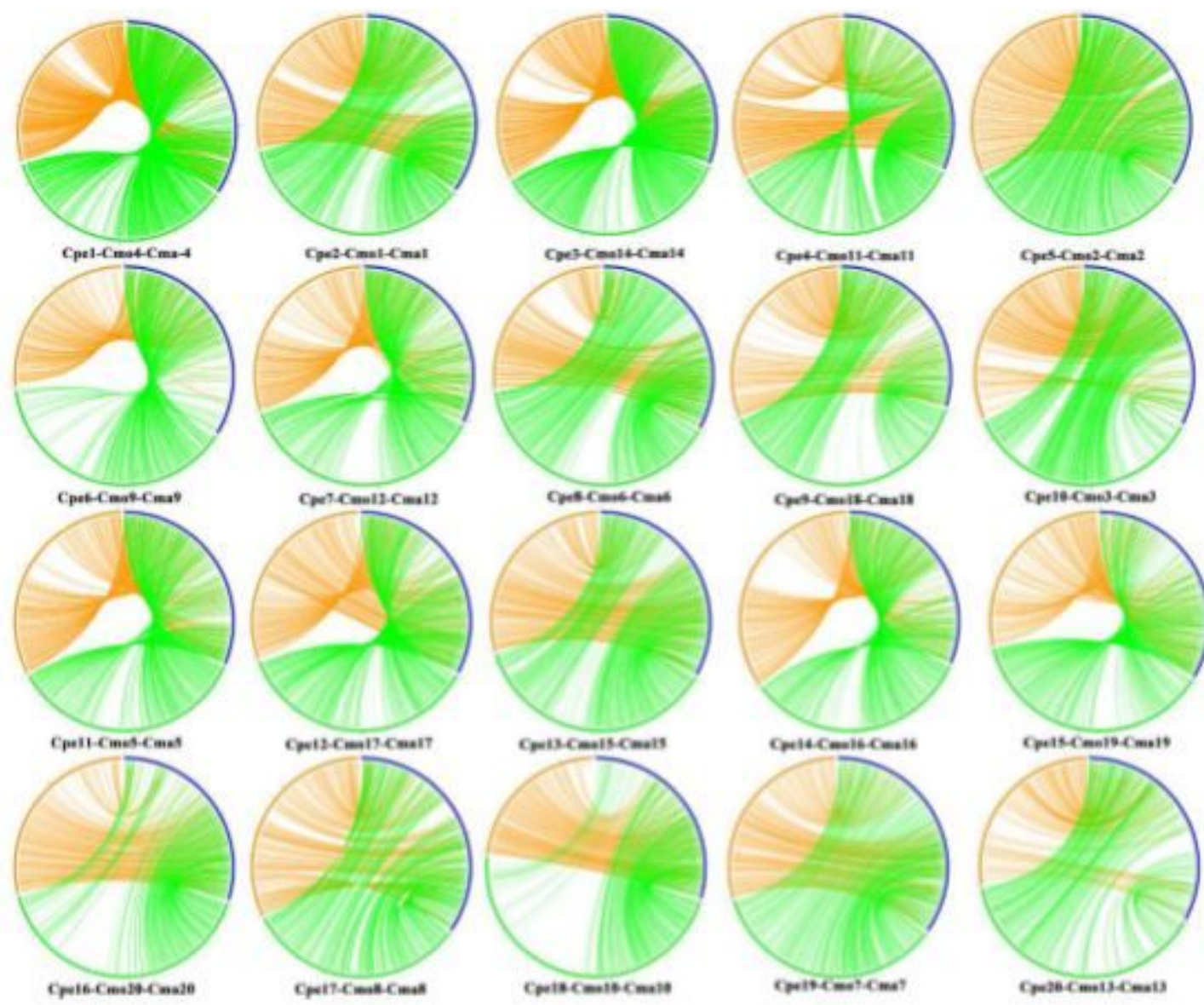

coesconitemat4
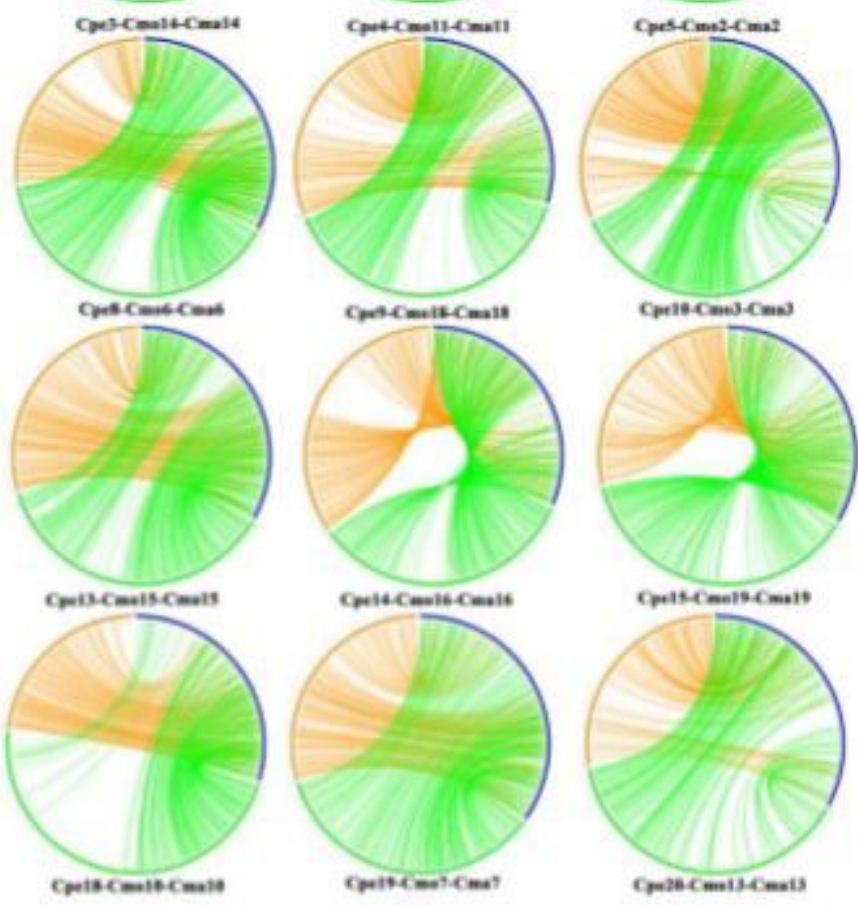

\section{Figure 4}

Chromosome synteny of C.pepo (blue) with C.moschata (green) and C.maxima (yellow). The physical positions of chromosomes of each crop in the figure are arranged clockwise. Chromosome synteny between C.pepo and C.moschata is based on 14276 cross-species markers; synteny between C.pepo and C.maxima is based on 10655 cross-species markers. Cpe1-Cpe20 represent C.pepo-twenty chromosomes, Cmo1-Cmo20 represent C.moschata chromosomes and $\mathrm{Cma1-Cma20}$ represent C.maxima chromsomes. The syntenic relationship between C.pepo and C.moschata are connected with the green color lines, and the syntenic relationship between C.pepo and C.maxima are connected with the yellow color lines. 

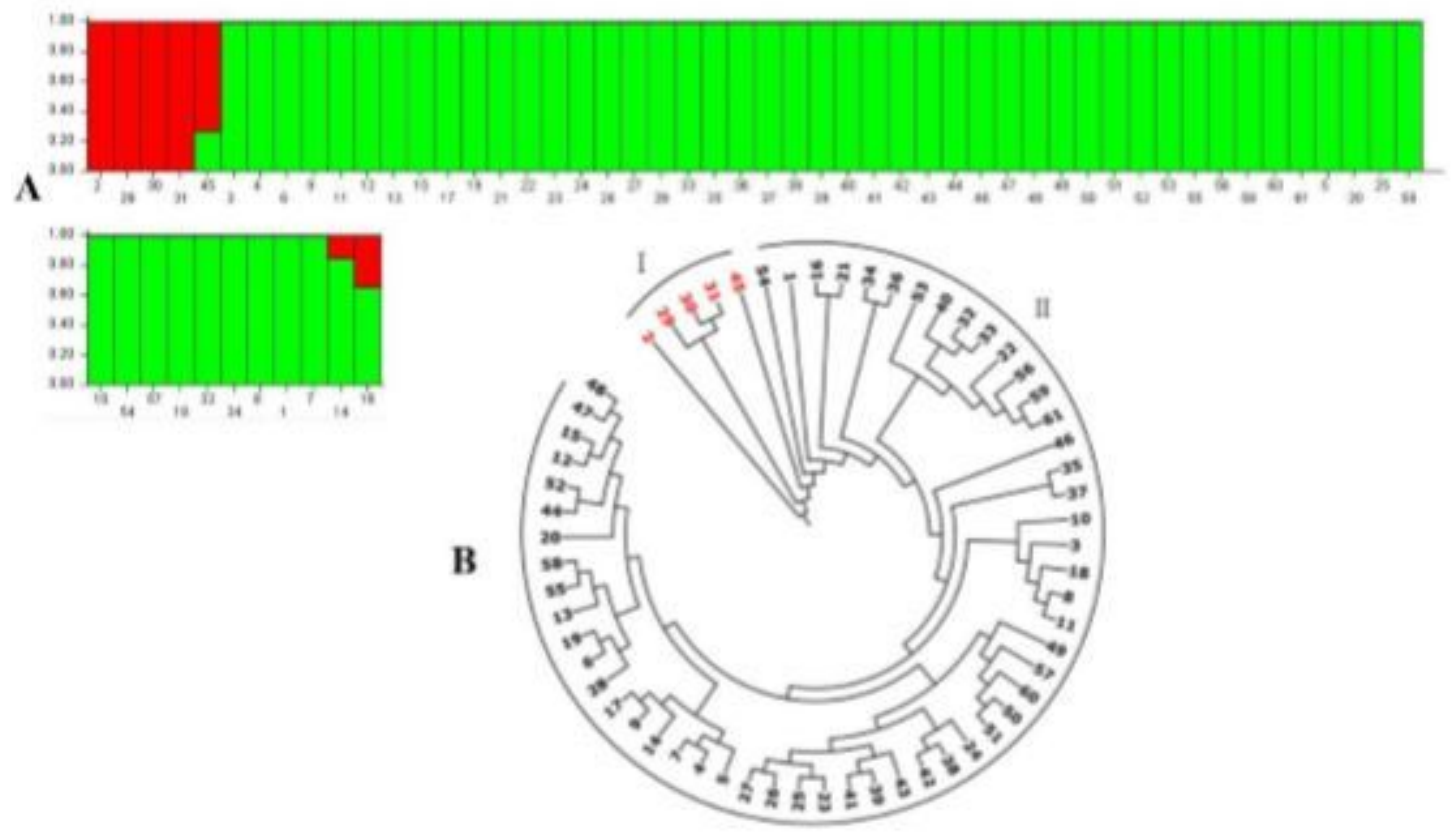

Figure 5

The UPGMA phylogenetic tree of the 61 accessions based on SSR markers. The phylogenetic analysis showed 61 accessions were classified into two groups: group I and II (A). Population structure of 61 accessions in C.pepo by Model-based analysis. Scale of $Y$ axis represents the percent of genetic components, and the $\mathrm{X}$ axis represents the different materials. Group I and II corresponds to the predefined phylogenetic tree (B). 